\title{
Matrix Analysis of Discontinuities in Nonreciprocal Waveguides: Analytical Description for Magnetooptical Slab Waveguides
}

\author{
Borja Sepúlveda and Laura M. Lechuga
}

\begin{abstract}
A new analytical formalism to study the effect of discontinuities in a nonreciprocal waveguide has been developed. The analysis is based on 1) the general orthogonality relation obtained from the reciprocity theorem, 2) the modal expansion of the transverse electromagnetic fields within the waveguide, and 3 ) the continuity of the tangential components of such electromagnetic fields at both sides of the discontinuity. The formalism is presented in a matrix form, which allows the treatment of several discontinuities as a simple summation and product of coupling and propagation matrices. The matrix formalism is developed for magnetooptic waveguides in the three different orientations of the magnetization, and, within this context, two practical applications of magnetooptic isolators are studied.
\end{abstract}

Index Terms-Isolators, magnetooptic waveguides, matrix analysis of discontinuities, nonreciprocal waveguides.

\section{INTRODUCTION}

$\mathbf{T}$ HE USE of nonreciprocal layers is becoming increasingly interesting for the development of integrated optical devices such as isolators [1]-[9], circulators, or modulators [10]-[12]. Before fabrication, these devices require a careful design to optimize their performances. For this purpose, different formalisms have been used to study the propagation of the light for planar [13] or more complicated structures [14]-[19]. Nevertheless, these devices are usually interconnected to other active or passive components such as waveguides or divisors. Therefore, the behavior of the light at the interconnections of the different components should be taken into account in the design of the whole integrated optical circuit. Great effort has been made to study the propagation of light through such discontinuities in isotropic media [20]-[23], where the modes of the guiding structures form an orthogonal basis. However, in the case of arbitrary anisotropic media, the modes supported by the guiding media do not form, in general, an orthogonal basis. Thus, the aim of this article is to study in detail the coupling between the light modes at step discontinuities when at least one of the media is anisotropic and nonreciprocal, i.e., when its dielectric tensor is nonsymmetric. This new analysis

Manuscript received November 5, 2003; revised April 27, 2003. This work was supported in part by the Ministerio de Ciencia y Tecnología under Project MAT2002-04484-C03-01 and in part by the Consejo Superior de Investigaciones Científicas (CSIC) under the I3P program.

The authors are with the Biosensors Group of the Microelectronics National Centre (IMM-CNM), CSIC, 28760 Madrid, Spain (e-mail: borja@imm.cnm.csic.es; website: http://www.imm.cnm.csic.es/biosensores/ home.html).

Digital Object Identifier 10.1109/JLT.2004.831094 for nonreciprocal waveguides is based on the following three different statements:

1) the general orthogonality relation obtained from the reciprocity theorem [24], [25];

2) the modal expansion of the electromagnetic fields inside the guiding structures;

3) the continuity of the tangential components of the electromagnetic fields at the interface between two different guiding media.

In addition, the analysis can be expressed in a matrix form, allowing the study of subsequent discontinuities as a summation and product of coupling and propagation matrices.

This paper is organized as follows. Section II describes the general orthogonality relation between modes in nonreciprocal waveguides and the modal expansion of the electromagnetic fields. In Section III, the continuity of the tangential components at step discontinuities is used to establish the general relations between the modes for arbitrary nonreciprocal media. Finally, Sections IV and V present the developed formalism in the case of magnetooptical waveguides for the three different orientations of the magnetization together with two practical applications for magnetooptic isolators.

\section{ORTHOGONALITY RELATION AND MODAL EXPANSION}

A general method to describe any electromagnetic field distribution in a waveguide can be done using the electromagnetic modes of the guiding structure, which are solutions of the Maxwell equations of the form

$$
\begin{aligned}
& E(x, y, z)=E_{n}(y, z) e^{i\left(k_{0} N_{n} x-\omega t\right)} \\
& H(x, y, z)=H_{n}(y, z) e^{i\left(k_{0} N_{n} x-\omega t\right)}
\end{aligned}
$$

where $E_{n}$ and $H_{n}$ are, in general, vectorial functions with three components, $k_{0}$ is the wave vector in vacuum, $N_{n}$ is the effective propagation index, and $\omega$ stands for the frequency of the light. We have assumed that the light is propagating along the $x$ direction and the waveguide is invariant in such direction.

In the case of isotropic waveguides, the modes are orthogonal to each other, and the orthogonality relation is given by the expression

$$
\iint_{-\infty}^{\infty} E_{T n}(y, z) \times H_{T \nu}(y, z) d y d z=0, \quad \nu \neq n
$$

where the $T$ subscript denotes the transversal field components, i.e., the $y$ and $z$ components. Such orthogonality relation allows 
the expression of any transversal field distribution as a superposition of modes of the waveguide. In contrast, in nonreciprocal waveguides, the modes are not, in general, orthogonal to each other and the previously described relation between modes is not valid. However, by using the reciprocity theorem [24], [25], a general orthogonality relation between any pair of modes, for reciprocal or nonreciprocal waveguides, can be established [26] as shown in (3) at the bottom of the page, where $E_{T n}$ and $H_{T n}$ are the transversal component of the electric and magnetic fields of the $n^{t h}$ mode of the waveguide, respectively. On the other hand, the prime mode represents the $\nu$ th mode of the structure in a situation in which the dielectric tensors of the nonreciprocal media are transposed (for magnetooptical materials, the dielectric tensor is transposed when the magnetization is reversed). In such a situation, the waveguide will be denoted as the complementary waveguide. In the case of reciprocal waveguides, the dielectric tensor is symmetric, and therefore, the prime mode denotes as well the $\nu$ th mode of the normal waveguide. In expression (3), $N_{n}$ and $N_{\nu}^{\prime}$ are the effective propagation indexes of the $n$th and $\nu$ th modes of the normal and the complementary waveguides, respectively, and $u_{x}$ represents the unitary vector in the $x$ direction (see Fig. 1).

Expression (3) can be used to extract a particular mode of a modal expansion only if the integral is not zero, that is, if $N_{n}=-N_{\nu}^{\prime}$ is fulfilled. If the $n$th and $\nu$ th modes are traveling in the same direction in the $x$ axis, the relation $N_{n}+N_{\nu}^{\prime}$ is never zero, forcing the integral to vanish. Therefore, both modes must travel in opposite directions, and the relation (3) can be rewritten as (4), shown at the bottom of the page, where the + and - refer to modes traveling in the positive or negative direction of the $x$ axis, respectively. As a consequence, for reciprocal or nonreciprocal media, (4) establishes that the integral must be zero, unless

$$
N_{n}^{+}=-N_{\nu}^{-\prime}
$$

This means that the waveguide must be bidirectional for reciprocal systems. However, in nonreciprocal systems, the waveguide must be bidirectional with respect to the complementary waveguide (for example, with its magnetization reversed in magnetooptical waveguides). A condition for a waveguide to be bidirectional is to have, at least, one symmetry operation that interchanges the + and - directions of the $x$ axis [26].

As it was mentioned previously, any field distribution of the transversal components of the electromagnetic fields can be expressed as a modal expansion of the modes of the guiding structure. Depending on the selected boundary conditions, it can be differentiated between closed and open waveguides. Then, in the case of closed waveguides, an arbitrary transversal field can

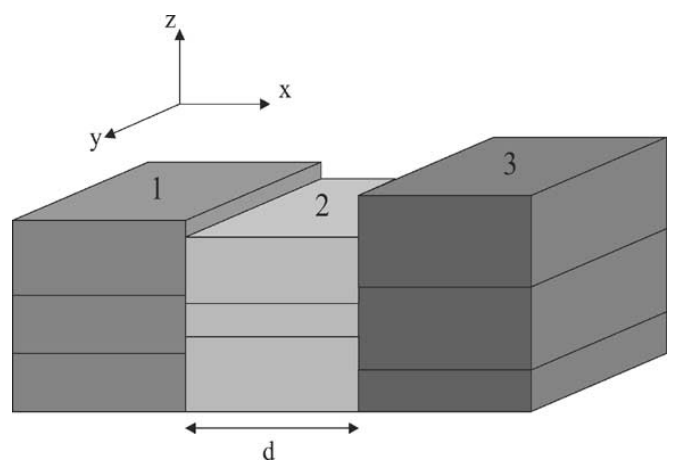

Fig. 1. Three layered waveguides separated by two step discontinuities. The light propagates in the $x$ direction.

be written as a sum over the infinite set of discrete modes supported by the waveguide

$$
\begin{aligned}
\mathcal{E}_{T} & =\sum_{n=0}^{\infty}\left(a_{n} \cdot E_{T n}^{+}(y, z) e^{i k_{0} N_{n}^{+} x}+b_{n} \cdot E_{T n}^{-}(y, z) e^{i k_{0} N_{n}^{-} x}\right) \\
\mathcal{H}_{T} & =\sum_{n=0}^{\infty}\left(a_{n} \cdot H_{T n}^{+}(y, z) e^{i k_{0} N_{n}^{+} x}+b_{n} \cdot H_{T n}^{-}(y, z) e^{i k_{0} N_{n}^{-} x}\right) .
\end{aligned}
$$

For open waveguides, the expansion must be carried out for the finite set of discrete modes as well as for the continuous spectrum of radiative modes of the waveguide. As a consequence, the transverse fields will be expressed as

$$
\begin{aligned}
\mathcal{E}_{T} & =\sum_{n=0}^{M}\left(a_{n} \cdot E_{T n}^{+}(y, z) e^{i k_{0} N_{n}^{+} x}+b_{n} \cdot E_{T n}^{-}(y, z) e^{i k_{0} N_{n}^{-} x}\right)+E_{\mathrm{rad}} \\
\mathcal{H}_{T} & =\sum_{n=0}^{M}\left(a_{n} \cdot H_{T n}^{+}(y, z) e^{i k_{0} N_{n}^{+} x}+b_{n} \cdot H_{T n}^{-}(y, z) e^{i k_{0} N_{n}^{-} x}\right)+H_{\mathrm{rad}}
\end{aligned}
$$

where $E_{\text {rad }}$ and $H_{\text {rad }}$ represent the continuous part of the modal spectrum.

If the condition (5) is fulfilled and the modes are not normalized, using the modal expansion and the orthogonality relation given by (4), the following expressions can be found:

$$
\begin{aligned}
& \iint\left(E_{T \nu}^{-\prime} \times H_{T n}^{+}-E_{T n}^{+} \times H_{T \nu}^{-\prime}\right) \cdot u_{x} d y d z=\chi_{n}^{+-} \\
& \iint\left(E_{T \nu}^{+\prime} \times H_{T n}^{-}-E_{T n}^{-} \times H_{T \nu}^{+\prime}\right) \cdot u_{x} d y d z=\chi_{n}^{-+}
\end{aligned}
$$

where $\chi_{n}^{+-}$and $\chi_{n}^{-+}$are the normalization constants of the $n$th mode and the integral is over the transversal plane $y z$. Hereafter,

$$
\left(N_{\nu}^{\prime}+N_{n}\right) \cdot \iint\left(E_{T \nu}^{\prime}(y, z) \times H_{T n}(y, z)-E_{T n}(y, z) \times H_{T \nu}^{\prime}(y, z)\right) \cdot u_{x} d y d z=0
$$

$$
\left(N_{\nu}^{-\prime}+N_{n}^{+}\right) \cdot \iint\left(E_{T \nu}^{-\prime}(y, z) \times H_{T n}^{+}(y, z)-E_{T n}^{+}(y, z) \times H_{T \nu}^{-\prime}(y, z)\right) \cdot u_{x} d y d z=0
$$


we will assume that the modes of the modal expansion are normalized.

Providing the condition (5) is again satisfied, the coefficients of the modes within the modal expansion can be obtained through (4), as can be seen in

$$
\begin{aligned}
& a_{n} e^{i k_{0} N_{n}^{+} x}=\iint\left(E_{T \nu}^{-\prime} \times \mathcal{H}_{T}-\mathcal{E}_{T} \times H_{T \nu}^{-\prime}\right) \cdot u_{x} d y d z \\
& b_{n} e^{i k_{0} N_{n}^{-} x}=\iint\left(E_{T \nu}^{+\prime} \times \mathcal{H}_{T}-\mathcal{E}_{T} \times H_{T \nu}^{+\prime}\right) \cdot u_{x} d y d z .
\end{aligned}
$$

\section{TRANSMISSION AND REFLECTION THROUGH STEP DISCONTINUITIES}

The system under study is shown in Fig. 1. Such a system is composed of three layered waveguides, which are separated by two step discontinuities. The first and third waveguides are semi-infinite and the light comes from the first one. We consider the general case in which all the media are nonreciprocal. If $\left(E_{T m}^{1}, H_{T m}^{1}\right),\left(E_{T n}^{2}, H_{T n}^{2}\right),\left(E_{T s}^{3}, H_{T s}^{3}\right)$ are the set of modes supported by the first, second, and third guiding media, respectively, and $\left(E_{T \mu}^{1 \prime}, H_{T \mu}^{1 \prime}\right),\left(E_{T \nu}^{2 \prime}, H_{T \nu}^{2 \prime}\right),\left(E_{T \sigma}^{3 \prime}, H_{T \sigma}^{3 \prime}\right)$ their respective complementary modes, which satisfy the bidirectionality conditions between the normal and complementary waveguides given by

$$
\begin{aligned}
& N_{m}^{1+}=N_{\mu}^{1-\prime} \\
& N_{n}^{2+}=N_{\nu}^{2-\prime} \\
& N_{s}^{3+}=N_{\sigma}^{3-\prime}
\end{aligned}
$$

then, using (9) and the continuity of the tangential components of the electric and magnetic fields at the first interface, we can obtain (11), shown at the bottom of the page. These equations can be written in a matrix form as

$$
\left(\begin{array}{l}
a_{n}^{2} \\
b_{n}^{2}
\end{array}\right)=\sum_{m}\left(\begin{array}{ll}
k_{\nu m}^{11} & k_{\nu m}^{12} \\
k_{\nu m}^{21} & k_{\nu m}^{22}
\end{array}\right)\left(\begin{array}{c}
a_{m}^{1} \\
b_{m}^{1}
\end{array}\right)
$$

where

$$
\begin{aligned}
k_{\nu m}^{11} & =\iint\left(E_{\nu}^{2-\prime} \times H_{m}^{1+}-E_{m}^{1+} \times H_{\nu}^{2-\prime}\right) \cdot u_{x} d y d z \\
k_{\nu m}^{12} & =\iint\left(E_{\nu}^{2-\prime} \times H_{m}^{1-}-E_{m}^{1-} \times H_{\nu}^{2-\prime}\right) \cdot u_{x} d y d z \\
k_{\nu m}^{21} & =\iint\left(E_{\nu}^{2+\prime} \times H_{m}^{1+}-E_{m}^{1+} \times H_{\nu}^{2+\prime}\right) \cdot u_{x} d y d z \\
k_{\nu m}^{22} & =\iint\left(E_{\nu}^{2+\prime} \times H_{m}^{1-}-E_{m}^{1-} \times H_{\nu}^{2+\prime}\right) \cdot u_{x} d y d z .
\end{aligned}
$$

In a contracted form, (12) can be expressed by

$$
\psi_{n}^{2}=\sum_{m} \mathbf{K}_{\nu m}^{21} \psi_{m}^{1}
$$

The $\mathbf{K}_{\nu m}^{21}$ matrices describe the coupling of the $m$ modes of the first waveguide into the mode $n$ of the second.

If we now consider the interface of the second and third waveguides, we can find (15), shown at the bottom of the page, or in matrix form

$$
\left(\begin{array}{c}
a_{s}^{3} \\
0
\end{array}\right)=\sum_{n}\left(\begin{array}{ll}
k_{\sigma n}^{11} & k_{\sigma n}^{12} \\
k_{\sigma n}^{21} & k_{\sigma n}^{22}
\end{array}\right)\left(\begin{array}{cc}
e^{i k_{0} N_{n}^{2+} d} & 0 \\
0 & e^{i k_{0} N_{n}^{2-} d}
\end{array}\right)\left(\begin{array}{c}
a_{n}^{2} \\
b_{n}^{2}
\end{array}\right)
$$

$$
\begin{aligned}
& a_{n}^{2}=\iint\left(E_{T \nu}^{2-\prime} \times \sum_{m}\left(a_{m}^{1} \cdot H_{T m}^{1+}(y, z)+b_{m}^{1} \cdot H_{T m}^{1-}(y, z)\right)-\sum_{m}\left(a_{m}^{1} \cdot E_{T m}^{1+}(y, z)+b_{m}^{1} \cdot E_{T m}^{1-}(y, z)\right) \times H_{T \nu}^{2-\prime}\right) \cdot u_{x} d y d z \\
& b_{n}^{2}=\iint\left(E_{T \nu}^{2+\prime} \times \sum_{m}\left(a_{m}^{1} \cdot H_{T m}^{1+}(y, z)+b_{m}^{1} \cdot H_{T m}^{1-}(y, z)\right)-\sum_{m}\left(a_{m}^{1} \cdot E_{T m}^{1+}(y, z)+b_{m}^{1} \cdot E_{T m}^{1-}(y, z)\right) \times H_{T \nu}^{2+\prime}\right) \cdot u_{x} d y d z .
\end{aligned}
$$

$$
\begin{aligned}
a_{s}^{3}= & \iint\left(E_{T \sigma}^{3-\prime} \times \sum_{n}\left(a_{n}^{2} \cdot H_{T n}^{2+}(y, z) e^{i k_{0} N_{n}^{2+} d}+b_{n}^{2} \cdot H_{T n}^{2-}(y, z) e^{i k_{0} N_{n}^{2-} d}\right)\right. \\
& \left.-\sum_{n}\left(a_{n}^{2} \cdot E_{T n}^{2+}(y, z) e^{i k_{0} N_{n}^{2+} d}+b_{n}^{2} \cdot E_{T n}^{2-}(y, z) e^{i k_{0} N_{n}^{2-} d}\right) \times H_{T \sigma}^{3-\prime}\right) \cdot u_{x} d y d z \\
0= & \iint\left(E_{T \sigma}^{3+\prime} \times \sum_{n}\left(a_{n}^{2} \cdot H_{T n}^{2+}(y, z) e^{i k_{0} N_{n}^{2+} d}+b_{n}^{2} \cdot H_{T n}^{2-}(y, z) e^{i k_{0} N_{n}^{2-} d}\right)\right. \\
& \left.-\sum_{n}\left(a_{n}^{2} \cdot E_{T n}^{2+}(y, z) e^{i k_{0} N_{n}^{2+} d}+b_{n}^{2} \cdot E_{T n}^{2-}(y, z) e^{i k_{0} N_{n}^{2-} d}\right) \times H_{T \sigma}^{3+\prime}\right) \cdot u_{x} d y d z
\end{aligned}
$$


where

$$
\begin{aligned}
k_{\sigma n}^{11} & =\iint\left(E_{\sigma}^{3-\prime} \times H_{n}^{2+}-E_{n}^{2+} \times H_{\sigma}^{3-\prime}\right) \cdot u_{x} d y d z \\
k_{\sigma n}^{12} & =\iint\left(E_{\sigma}^{3-\prime} \times H_{n}^{2-}-E_{n}^{2-} \times H_{\sigma}^{3-\prime}\right) \cdot u_{x} d y d z \\
k_{\sigma n}^{21} & =\iint\left(E_{\sigma}^{3+\prime} \times H_{n}^{2+}-E_{n}^{2+} \times H_{\sigma}^{3+\prime}\right) \cdot u_{x} d y d z \\
k_{\sigma n}^{22} & =\iint\left(E_{\sigma}^{3+\prime} \times H_{n}^{2-}-E_{n}^{2-} \times H_{\sigma}^{3+\prime}\right) \cdot u_{x} d y d z .
\end{aligned}
$$

As in the case of the first interface, this equation can be written in a contracted form as

$$
\psi_{s}^{3}=\sum_{n} \mathbf{K}_{\sigma n}^{32} \mathbf{P}_{n}^{2} \psi_{n}^{2} .
$$

The introduction of (14) in (18) allows the relation of the modes of the first and third media:

$$
\psi_{s}^{3}=\sum_{n} \sum_{m} \mathbf{K}_{\sigma n}^{32} \mathbf{P}_{n}^{2} \mathbf{K}_{\nu m}^{21} \psi_{m}^{1}
$$

In this equation, the $\mathbf{K}_{\sigma n}^{32}$ matrices represent the coupling of the $n$ modes of the second waveguide into the $s$ mode of the third guiding medium, and $\mathbf{P}_{n}^{2}$ stands for the propagation of the $n$ mode along the distance $d$ of the second waveguide. The nonreciprocity of the media is taken into account within $\mathbf{P}_{n}^{2}$, since the propagation constants for the forward- and backwardtraveling modes are, in general, different $\left(N_{n}^{2+} \neq N_{n}^{2-}\right)$.

The nonreciprocity is also taken into account in the calculation of the normalization constants of the modes and coupling matrices, since the modes of the complementary waveguides whose effective propagation constants satisfy (10) are introduced in the calculations.

Once we have obtained the relations between the modes of the first and third waveguides, we are able to calculate the reflectivity and transmitivity of each mode through the structure. Equation (19) can be expressed as

$$
\begin{aligned}
\psi_{s}^{3} & =\sum_{m}\left(\sum_{n} \mathbf{K}_{\sigma n}^{32} \mathbf{P}_{n}^{2} \mathbf{K}_{\nu m}^{21}\right) \psi_{m}^{1}=\sum_{m} \mathbf{J}_{s m}^{31} \psi_{m}^{1} \\
\left(\begin{array}{c}
a_{s}^{3} \\
0
\end{array}\right) & =\sum_{m} \mathbf{J}_{s m}^{31}\left(\begin{array}{c}
a_{m}^{1} \\
b_{m}^{1}
\end{array}\right) .
\end{aligned}
$$

Then, the $\mathbf{J}_{s m}^{31}$ matrices connect the $m$ modes of the first waveguide with the $s$ mode of the third guiding medium, acting as a waveguide transfer matrix.

Generally, the incident coefficients $a_{m}^{1}$ will be known values and can be expressed as

$$
a_{m}^{1}=a \cdot x_{m}
$$

where

$$
0<\left|x_{m}\right|<1, \quad \sum_{m}\left|x_{m}\right|=1
$$

with $x_{m}$ representing the fraction of mode $m$ with respect to the total of incident modes.
If (21) is introduced into (20) and the result is divided by $a$, the next expression obtained is

$$
\left(\begin{array}{c}
t_{s} \\
0
\end{array}\right)=\sum_{m} \mathbf{J}_{s m}^{31}\left(\begin{array}{c}
x_{m} \\
r_{m}
\end{array}\right) \text {. }
$$

In this equation, $t_{s}$ represents the transmitivity of the mode $s$ of the third waveguide, and $r_{m}$ is the reflectivity of the $m$ mode of the first guiding medium. Considering all the $m$ and $s$ modes of the first and third waveguides, respectively, a set of linear equations is obtained. Its solution provides the transmitivities and reflectivities of the different modes.

The formalism can be extended to any number of discontinuities simply by adding and multiplying coupling and propagation matrices, as is done in (19). Assuming $N$ discontinuities, the modes at the $N+1$ guiding medium will be given by

$$
\psi_{s}^{N+1}=\sum_{m} \prod_{i=2}^{N}\left(\sum_{u} \mathbf{K}_{\varpi u}^{(i+1) i} \mathbf{P}_{u}^{i}\right) \mathbf{K}_{\nu m}^{21} \psi_{m}^{1} .
$$

\section{Discontinuities In Magnetooptic WaVEGuides}

We can apply the developed formalism to analyze the effect of discontinuities in magnetooptic waveguides for different orientations of the magnetization. Thus, we are going to consider the case of a magnetooptical waveguide, which is between two isotropic guiding media. The dielectric tensor of a magnetooptic material with the magnetization in an arbitrary direction is expressed by

$$
\boldsymbol{\varepsilon}=\left(\begin{array}{ccc}
\varepsilon_{11} & \varepsilon_{12} & \varepsilon_{13} \\
-\varepsilon_{12} & \varepsilon_{22} & -\varepsilon_{23} \\
-\varepsilon_{13} & \varepsilon_{23} & \varepsilon_{33}
\end{array}\right)
$$

As can be observed, this dielectric tensor is nonsymmetric, as is the case of the nonreciprocal media. The nondiagonal elements of the dielectric tensor have a linear dependence on the magnetization, while the dependence is quadratic for the diagonal terms. Therefore, when the magnetization is reversed, the dielectric tensor is transposed, that is, $\varepsilon(-M)=\varepsilon(M)^{t}$.

As a result, the magnetooptic complementary waveguide is obtained reversing the direction of the magnetization.

For simplicity, we consider open-slab waveguides, since analytical solutions can be found for these systems. Another simplification that can be made is to assume that the coupling of the radiative modes into guided modes at the discontinuities can be neglected. Consequently, the radiative terms in the modal expansions (7) can be eliminated. Such simplification can be carried out if the discontinuity between the waveguides is not very strong [20] and if the separation between discontinuities is much bigger than the wavelength of the light.

The configuration of the isotropic waveguides is chosen to obtain only two guided modes, the $\mathrm{TE}_{0}$ and the $\mathrm{TM}_{0}$. On the other hand, the magnetooptic waveguide also has two guided modes but they will not be, in general, TE or TM.

To calculate the transverse resonant condition $\left(N_{n}\right)$ and the field distribution of the modes $\left(E_{n}, H_{n}\right)$ in the magnetoopticand isotropic-layered waveguides, we can use a $4 \times 4$ matrix formalism developed by Vassell [13]. This formalism is used to ob- 
tain exact analytical solutions for arbitrary anisotropic-layered media and is based on the continuity of the tangential components of the electromagnetic fields at the interfaces of the layer stack ( $x$ and $y$ components).

In conclusion, this example deals with isotropic and magnetooptic waveguides, that is, reciprocal and nonreciprocal systems. Since their propagation properties and symmetries are different, both systems will be treated separately in the following subsections.

\section{A. Isotropic Waveguides}

Regarding the isotropic-layered waveguides (first and third waveguides), the guided solutions of the Maxwell equations can be grouped into TE or TM modes, and both polarizations are uncoupled. With regard to their symmetry, it can be shown that [26], [27]

$$
N_{m}^{+}=-N_{m}^{-} .
$$

Then, the forward- and backward-traveling modes have the same effective propagation constant, but with opposite signs, producing a bidirectional waveguide. On the other hand, the fields of the forward- and backward-traveling modes satisfy [26], [27]

$$
\begin{aligned}
E_{T m}^{+} & =E_{T m}^{-}=E_{T m} \\
E_{x m}^{+} & =-E_{x m}^{-}=E_{x m} \\
H_{T m}^{+} & =-H_{T m}^{-}=H_{T m} \\
H_{x m}^{+} & =H_{x m}^{-}=H_{x m} .
\end{aligned}
$$

The relations (25) and (26) simplify the orthogonality equation between modes (see (1)), as well as the modal expansion of the transversal components.

\section{B. Magnetooptic Waveguides}

The propagation properties of the light in a magnetooptic waveguide hinge on the direction of the applied magnetic field. Depending on the direction, three different configurations can be distinguished: transversal, longitudinal, and polar $(y, x$, and $z$ directions, respectively).

1) Transversal Configuration: In this configuration, the magnetization lays in the $y$ axis, and therefore, the dielectric tensors of the magnetooptical layers are represented by

$$
\boldsymbol{\varepsilon}=\left(\begin{array}{ccc}
\varepsilon_{11} & 0 & \varepsilon_{13} \\
0 & \varepsilon_{22} & 0 \\
-\varepsilon_{13} & 0 & \varepsilon_{11}
\end{array}\right)
$$

The elements $\varepsilon_{11}$ and $\varepsilon_{22}$ are different to take into account the quadratic magnetooptic effect. Within this configuration, the modes supported by the structure are also TE and TM, with both being uncoupled. Since the TE and TM modes are orthogonal, both polarizations can be treated separately.

In this configuration, the magnetization effects come from different elements of the dielectric tensor for each light polarization. The TE mode has only one component of the electric field $E_{y}$. Accordingly, the effect of the magnetization over this mode can only be owed to the quadratic magnetooptic coeffi- cient present within the $\varepsilon_{22}$ term. Since the effect is quadratic, a change in the sign of the magnetization does not modify the propagation properties of the guided light. As a consequence, the normal and the complementary waveguides are identical and the following relation is satisfied:

$$
N_{m}^{+}(\mathrm{TE})=-N_{m}^{-\prime}(\mathrm{TE})=-N_{m}^{-}(\mathrm{TE}) .
$$

Then, in the case of the TE modes, the waveguide is bidirectional and the propagation properties and symmetries of the isotropic systems, (25) and (26), are applicable. The effect of the magnetization on the propagation properties of the TE mode is a slight variation of the effective propagation constant $N_{m}$. Since such variation comes from the $\varepsilon_{22}$ element of the dielectric tensor, $N_{m}$ becomes a quadratic function of the magnetic field.

In contrast, the TM mode has two electric components $\left(E_{x}\right.$ and $E_{z}$ ), which are coupled by means of the nondiagonal element $\varepsilon_{13}$. In this situation, if the magnetization is reversed, the propagation properties are modified. Such behavior can be seen as a consequence of the nonreciprocity of the waveguide for the TM mode. Therefore, the forward- and backward-traveling modes do not satisfy (25) and (26). Besides, the propagation constants of the modes traveling in the + or - directions of the $x$ axis are different, i.e., $N_{m}^{+} \neq-N_{m}^{-}$.

However, owed to symmetry considerations, the following relation for the TM mode in the transversal configuration can be found [26]:

$$
N_{m}^{+}(\mathrm{TM})=-N_{m}^{-\prime}(\mathrm{TM})
$$

that is, the effective propagation constant of the forward mode corresponds to the propagation constant of the same backward mode, but for the complementary waveguide (with the magnetic field applied in the opposite direction). With regard to the symmetry of the electromagnetic fields, it is possible to correlate the forward modes of the normal waveguide and the backward modes of the complementary waveguide, finding

$$
\begin{aligned}
& E_{T m}^{+}=E_{T m}^{-\prime} \\
& H_{T m}^{+}=-H_{T m}^{-\prime} .
\end{aligned}
$$

Such relations simplify the formalism, since only the calculation of the forward and backward modes of the normal waveguide is necessary.

Although the dielectric elements involved in the magnetooptic effect are different in the TE and mode polarizations, the modification induced in the propagation properties of the modes is also a change in the effective propagation index $\left(N_{m}\right)$. However, this variation is linear for the TM mode, since it is due to the nondiagonal term $\varepsilon_{13}$.

In conclusion, the magnetization induces a reciprocal or nonreciprocal phase shift for the TE or TM polarizations, respectively.

If the waveguides are monomode for each polarization, (19), connecting the modes in the first and third waveguides, can be expressed as

$$
\psi_{i}^{3}=\mathbf{K}_{i i}^{32} \mathbf{P}_{i}^{2} \mathbf{K}_{i i}^{21} \psi_{i}^{1}
$$


where the subscript $i$ can denote indistinctly the TE or TM polarizations.

For each polarization, the $\mathbf{K}_{i i}^{21}$ matrix represents the coupling of the fundamental mode of the first medium into the only accessible guided mode of the second medium, $\mathbf{P}_{i}^{2}$ describes the reciprocal or nonreciprocal propagation along the second medium, and finally, the $\mathbf{K}_{i i}^{32}$ establishes the coupling of the fundamental mode of the second medium into the fundamental one of the third.

In this situation, (22) is transformed into

$$
\left(\begin{array}{c}
t_{i} \\
0
\end{array}\right)=\mathbf{J}_{i i}^{31}\left(\begin{array}{c}
1 \\
r_{i}
\end{array}\right)=\left(\begin{array}{ll}
j_{11} & j_{12} \\
j_{21} & j_{22}
\end{array}\right)\left(\begin{array}{c}
1 \\
r_{i}
\end{array}\right)
$$

and, therefore

$$
\begin{aligned}
& t_{i}=j_{11}-\frac{j_{12} j_{21}}{j_{22}} \\
& r_{i}=-\frac{j_{21}}{j_{22}} .
\end{aligned}
$$

The previous expressions give the reflectivity and transmitivity through the magnetooptical waveguide for each polarization separately. The effect of multiple reflections at the discontinuities is considered by the propagation matrix $\mathbf{P}_{i}^{2}$.

2) Longitudinal Configuration: In this configuration, the magnetization lays in the $x$ direction, resulting in the dielectric tensor for the magnetooptical layers

$$
\boldsymbol{\varepsilon}=\left(\begin{array}{ccc}
\varepsilon_{11} & 0 & 0 \\
0 & \varepsilon_{22} & -\varepsilon_{23} \\
0 & \varepsilon_{23} & \varepsilon_{22}
\end{array}\right) .
$$

Unlike the transversal configuration, the modes in the magnetooptic waveguide are not purely TE or TM, but a mixture of them, avoiding the separation of the different polarizations in the formalism.

Since the reflection symmetry is always satisfied for the slab waveguides and taking into consideration the axial character of the magnetization vector, it can be shown that the forward- and backward-guided modes exhibit the same propagation constant in this configuration [26], i.e.,

$$
N_{m}^{+}=-N_{m}^{-}=N_{m}
$$

while the fields satisfy

$$
\begin{aligned}
& E_{T m}^{+}=E_{T m}^{-}=E_{T m} \\
& H_{T m}^{+}=-H_{T m}^{-}=H_{T m} .
\end{aligned}
$$

These relations introduce some simplifications in the calculations and then only the calculation of the forward modes of the normal and complementary waveguide is necessary.

First, we consider the interface between the first and second waveguides, where the following relation between modes is fulfilled:

$$
\begin{aligned}
& \psi_{1}^{2}=\mathbf{K}_{1 \mathrm{TE}}^{21} \psi_{\mathrm{TE}}^{1}+\mathbf{K}_{1 \mathrm{TM}}^{21} \psi_{\mathrm{TM}}^{1} \\
& \psi_{2}^{2}=\mathbf{K}_{2 \mathrm{TE}}^{21} \psi_{\mathrm{TE}}^{1}+\mathbf{K}_{2 \mathrm{TM}}^{21} \psi_{\mathrm{TM}}^{1}
\end{aligned}
$$

neglecting again the coupling of the radiative modes into the guided modes of the second waveguide. Thus, the former equations represent the coupling of the TE and TM modes of the first isotropic waveguide into the first and second modes of the nonreciprocal waveguide.

Regarding the second discontinuity, the relation between the different modes is given by

$$
\begin{aligned}
\psi_{\mathrm{TE}}^{3} & =\mathbf{K}_{\mathrm{TE1}}^{32} \mathbf{P}_{1}^{2} \psi_{1}^{2}+\mathbf{K}_{\mathrm{TE} 2}^{32} \mathbf{P}_{2}^{2} \psi_{2}^{2} \\
\psi_{\mathrm{TM}}^{3} & =\mathbf{K}_{\mathrm{TM} 1}^{32} \mathbf{P}_{1}^{2} \psi_{1}^{2}+\mathbf{K}_{\mathrm{TM} 2}^{32} \mathbf{P}_{2}^{2} \psi_{2}^{2} .
\end{aligned}
$$

Then, (38) shows the coupling of the modes of the magnetooptical waveguide into the TE and TM modes of the third guiding medium. The propagation of the modes along the second guiding medium and the effect of multiple reflections is characterized by the propagation matrices $\mathbf{P}_{1}^{2}$ and $\mathbf{P}_{2}^{2}$. Therefore, an interference phenomenon takes place at the discontinuities since the nonreciprocal modes excite the TE and TM modes of the first and third waveguides.

Introducing the equations of the first discontinuity in (38) and rearranging terms, it can be found that

$$
\begin{aligned}
\psi_{\mathrm{TE}}^{3}= & \left(\mathbf{K}_{\mathrm{TE} 1}^{32} \mathbf{P}_{1}^{2} \mathbf{K}_{1 \mathrm{TE}}^{21}+\mathbf{K}_{\mathrm{TE} 2}^{32} \mathbf{P}_{2}^{2} \mathbf{K}_{2 \mathrm{TE}}^{21}\right) \cdot \psi_{\mathrm{TE}}^{1} \\
& +\left(\mathbf{K}_{\mathrm{TE} 1}^{32} \mathbf{P}_{1}^{2} \mathbf{K}_{1 \mathrm{TM}}^{21}+\mathbf{K}_{\mathrm{TE} 2}^{32} \mathbf{P}_{2}^{2} \mathbf{K}_{2 \mathrm{TM}}^{21}\right) \cdot \psi_{\mathrm{TM}}^{1} \\
\psi_{\mathrm{TM}}^{3}= & \left(\mathbf{K}_{\mathrm{TM} 1}^{32} \mathbf{P}_{1}^{2} \mathbf{K}_{1 \mathrm{TE}}^{21}+\mathbf{K}_{\mathrm{TM} 2}^{32} \mathbf{P}_{2}^{2} \mathbf{K}_{2 \mathrm{TE}}^{21}\right) \cdot \psi_{\mathrm{TE}}^{1} \\
& +\left(\mathbf{K}_{\mathrm{TM} 1}^{32} \mathbf{P}_{1}^{2} \mathbf{K}_{1 \mathrm{TM}}^{21}+\mathbf{K}_{\mathrm{TM} 2}^{32} \mathbf{P}_{2}^{2} \mathbf{K}_{2 \mathrm{TM}}^{21}\right) \cdot \psi_{\mathrm{TM}}^{1}
\end{aligned}
$$

These equations can also be expressed in a matrix form

$$
\left(\begin{array}{c}
\psi_{\mathrm{TE}}^{3} \\
\psi_{\mathrm{TM}}^{3}
\end{array}\right)=\left(\begin{array}{ll}
\mathbf{J}_{\mathrm{TETE}}^{31} & \mathbf{J}_{\mathrm{TETM}}^{31} \\
\mathbf{J}_{\mathrm{TMTE}}^{31} & \mathbf{J}_{\mathrm{TMTM}}^{31}
\end{array}\right)\left(\begin{array}{c}
\psi_{\mathrm{TE}}^{1} \\
\psi_{\mathrm{TM}}^{1}
\end{array}\right)
$$

The diagonal matrices represent the coupling between modes of the same polarization. In contrast, the nondiagonal matrices describe the conversion of the TE modes into the TM, and vice versa, owed to the effect of the longitudinal magnetization in the nonreciprocal waveguide.

The modes at the first discontinuity can be expressed as

$$
\begin{aligned}
a_{\mathrm{TE}}^{1} & =a \cos \theta \\
a_{\mathrm{TM}}^{1} & =a \sin \theta
\end{aligned}
$$

where $\theta$ represents polarization state at the first discontinuity.

Introducing the former relations in (42) and dividing by $a$ obtains

$$
\begin{aligned}
\left(\begin{array}{c}
t_{\mathrm{TE}}^{3} \\
0
\end{array}\right) & =\mathbf{J}_{\mathrm{TETE}}^{31}\left(\begin{array}{c}
\cos \theta \\
r_{\mathrm{TE}}^{1}
\end{array}\right)+\mathbf{J}_{\mathrm{TETM}}^{31}\left(\begin{array}{c}
\sin \theta \\
r_{\mathrm{TM}}^{1}
\end{array}\right) \\
\left(\begin{array}{c}
t_{\mathrm{TM}}^{3} \\
0
\end{array}\right) & =\mathbf{J}_{\mathrm{TMTE}}^{31}\left(\begin{array}{c}
\cos \theta \\
r_{\mathrm{TE}}^{1}
\end{array}\right)+\mathbf{J}_{\mathrm{TMTM}}^{31}\left(\begin{array}{c}
\sin \theta \\
r_{\mathrm{TM}}^{1}
\end{array}\right) .
\end{aligned}
$$

As a consequence, the polarization state of the transmitted light at the second discontinuity is given by

$$
\Theta_{t}=\arctan \frac{t_{\mathrm{TM}}^{3}}{t_{\mathrm{TE}}^{3}}
$$

while, for the reflected light, it is given by

$$
\Theta_{r}=\arctan \frac{r_{\mathrm{TM}}^{1}}{r_{\mathrm{TE}}^{1}} .
$$

The propagation constants of the modes in the magnetooptic waveguide are in general different, inducing a birefringence effect. Therefore, $\Theta_{t}$ and $\Theta_{r}$ will be complex numbers, whose 
real part will represent the rotation state of the light, while the imaginary part will correspond to the ellipticity.

As a conclusion, the magnetization induces a rotation of the polarization of the light traveling within the waveguide, and this effect depends on the nondiagonal term of the dielectric tensor $\varepsilon_{23}$. However, such rotation can be disturbed as a consequence of the birefringence of the modes in the nonreciprocal waveguide.

3) Polar Configuration: The polar configuration has the magnetization oriented in the $z$ direction and a dielectric tensor given by

$$
\boldsymbol{\varepsilon}=\left(\begin{array}{ccc}
\varepsilon_{11} & \varepsilon_{12} & 0 \\
-\varepsilon_{12} & \varepsilon_{11} & 0 \\
0 & 0 & \varepsilon_{33}
\end{array}\right)
$$

As in the case of the longitudinal configuration, the modes of the waveguide are not TE or TM. In this configuration, the magnetization is perpendicular to the direction of propagation of the light and, therefore, exhibits the same symmetry relations of the transversal configuration [26], given by (29) and (30). Such behavior is due to the axial character of the magnetic-field vector. In this direction of the magnetization, however, another symmetry relation $N_{m}^{+}=-N_{m}^{-}=N_{m}$ is satisfied.

Since the modes of the waveguide are not TE or TM, all the formalism that correlates the modes at each interface can be described identically to the case of the longitudinal configuration [(37)-(44)] but considering that the propagation and coupling matrices satisfy different symmetry relations.

\section{Practical Applications}

Typical applications of the nonreciprocal devices are isolators or circulators. Such commercial devices can be found nowadays in bulk form. There is a growing interest to develop these kinds of devices in integrated versions, because then they can be compatible with fiber optics, the magnetic fields required are low, and the production costs can be reduced. In the case of isolators, three different configurations can be found to achieve the isolation: a $45^{\circ}$ nonreciprocal rotation in the longitudinal configuration [1]-[4], and $\pi / 2$ nonreciprocal phase shifts in the transversal [7], [8] and polar [9] configurations, for the TM and TE modes, respectively. The most commonly used magnetooptic materials in the field of integrated optics are the yttrium iron garnets (YIG), as they present very good transparency in the near infrared. Recently, the study of the growth conditions of polycrystalline magnetic garnets over amorphous substrates such as $\mathrm{SiO}_{2}$ has begun, obtaining similar magnetooptic properties as the monocrystalline layers [30], [31]. Such studies are quite interesting since they allow the combination of the magnetooptical properties of the garnets with the advantages of the microelectronic technology. Within this context, an example of the developed formalism for a practical application as an isolator based on nonreciprocal phase shifts will be presented. Another possibility to join the magnetooptical waveguides and the silicon technology can be achieved via the hybrid integration [4]. As a consequence, the formalism will be used to simulate a hybridly integrated isolator in the longitudinal configuration.

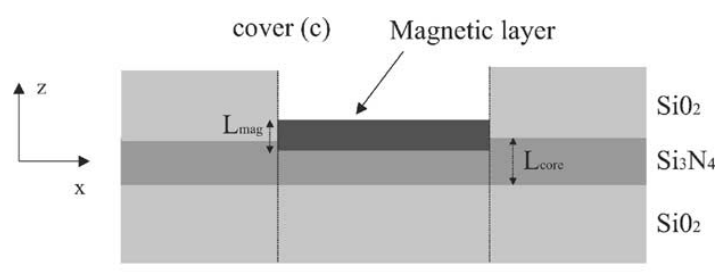

substrate (s)

Fig. 2. Waveguide structure for an isolator based in a nonreciprocal phase shift.

\section{A. Isolator Based on Nonreciprocal Phase Shifts}

The changes induced by the magnetization in the effective propagation index can be used to develop an isolator in an interferometric configuration, as in an integrated Mach-Zehnder interferometer (MZI) [7], [8]. If the interferometer is asymmetric with a reciprocal $\pi / 2$ phase difference between branches, a $\pi / 2$ nonreciprocal phase shift induced by the magnetooptic medium will cause a constructive interference for the forward-traveling light and destructive for the backreflected. The structure that will be studied is depicted in Fig. 2.

The first and third guiding media are two monomode waveguides with an $\mathrm{Si}_{3} \mathrm{~N}_{4}$ core and $\mathrm{SiO}_{2}$ claddings. The magnetooptic waveguide is composed by a Cerium-substituted YIG (Ce:YIG) layer deposited over an $\mathrm{Si}_{3} \mathrm{~N}_{4}$ layer. Different thickness will be chosen in order to optimize the magnetooptic effect, as well as the optical absorption and the radiation losses at the discontinuities. The refractive indexes of the $\mathrm{SiO}_{2}$ and $\mathrm{Si}_{3} \mathrm{~N}_{4}$ are 1.46 and 2.00, respectively. On the other hand, the dielectric constants of the Ce:YIG layer are $\varepsilon_{x x}=4.84+i 1.710^{-4}$ and $\varepsilon_{x z}=i 0.008$ at a wavelength of $1500 \mathrm{~nm}$ [6]. First, we will look for the configuration of the magnetooptic waveguide that maximizes the nonreciprocal phase shift without considering the radiation losses at the discontinuities.

Fig. 3(a) represents the propagation distance necessary to obtain a $\pi / 2$ nonreciprocal phase shift $\left(L_{\pi / 2}\right)$ as a function of the Ce:YIG layer thickness, for different thickness of the $\mathrm{Si}_{3} \mathrm{~N}_{4}$ layer, where $L_{\pi / 2}$ is given by

$$
L_{\pi / 2}=\frac{\lambda}{\left(4 \operatorname{Re}\left\lfloor N_{\mathrm{TM}}^{+}-N_{\mathrm{TM}}^{-}\right\rfloor\right)}
$$

with $\lambda$ being the wavelength of the light.

Conversely, the intensity found after the $L_{\pi / 2}$ propagation is shown in Fig. 3(b). With both graphs, it is possible to select the thickness of the layers to achieve the lowest absorption or the shortest propagation distance. In the first case, the optimum configuration is obtained for a $\mathrm{Si}_{3} \mathrm{~N}_{4}$ thickness of $300 \mathrm{~nm}$ and a Ce:YIG thickness of $150 \mathrm{~nm}$. In contrast, a 280-nm garnet layer deposited over a $300-\mathrm{nm} \mathrm{Si}_{3} \mathrm{~N}_{4}$ layer is necessary to minimize the propagation length.

Once the coupling with the first and third waveguides is introduced, the thickness of the $\mathrm{Si}_{3} \mathrm{~N}_{4}$ core layer of such waveguides is adjusted to optimize the coupling losses at the discontinuities, using (31)-(33).

For the first structure, with a 150-nm Ce:YIG layer, Fig. 4(a) shows that the highest transmitivity is achieved with a 340-nmthick $\mathrm{Si}_{3} \mathrm{~N}_{4}$ core layer in the first and third waveguides. For the second configuration, however, the optimal $\mathrm{Si}_{3} \mathrm{~N}_{4}$ thickness is 

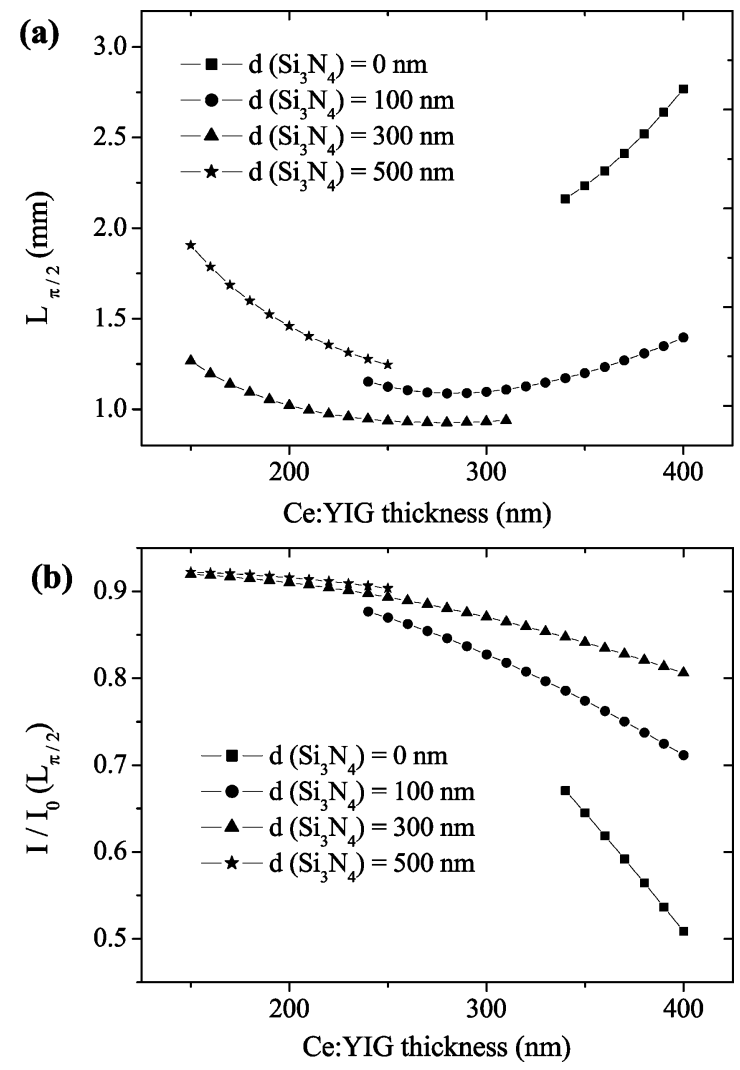

Fig. 3. (a) Propagation distance required to obtain a $\pi / 2$ nonreciprocal phase shift $\left(L_{\pi / 2}\right)$ as a function of the thickness of the garnet layer, for different thickness of the $\mathrm{Si}_{3} \mathrm{~N}_{4}$ layer. (b) Light intensity obtained after the $L_{\pi / 2}$ propagation distance.

$500 \mathrm{~nm}$ [Fig. 4(b)]. In both cases, the optimal configurations minimize the reflectivity at the discontinuities, preventing the problems of the backreflected light.

In conclusion, the isolation can be achieved for lengths of the magnetooptical area shorter than $1 \mathrm{~mm}$, with transmitivities better than $80 \%$, allowing the fabrication of very compact devices. Besides, in this type of structures, the top $\mathrm{SiO}_{2}$ layer acts as a protecting mask, allowing the direct deposition of the magnetooptic material in the desired regions. Therefore, such a structure can facilitate the fabrication process of the device.

\section{B. Hybrid Isolator Based on a $45^{\circ}$ Nonreciprocal Rotation}

Another method to obtain the isolation from the backreflected light is the use of a nonreciprocal rotation of $45^{\circ}$ in the longitudinal configuration. The nonreciprocal rotator is hybridly integrated within a silica integrated optical circuit. The required structure to achieve the isolation is described in [5] and is composed by the $45^{\circ}$ nonreciprocal rotator, a half-wave plate, and two polarizers. As a consequence, an incident TE mode traveling in the forward direction maintains its polarization at the output, while the backward-traveling light is rotated $90^{\circ}$ and blocked with the input polarizer.

We are going to study the effects of the discontinuities introduced by the hybrid integration. The magnetooptic waveguide is formed by three YIG layers whose optical properties are the same as [5] and whose thickness is $1.8 \mu \mathrm{m}$ (see Fig. 5).
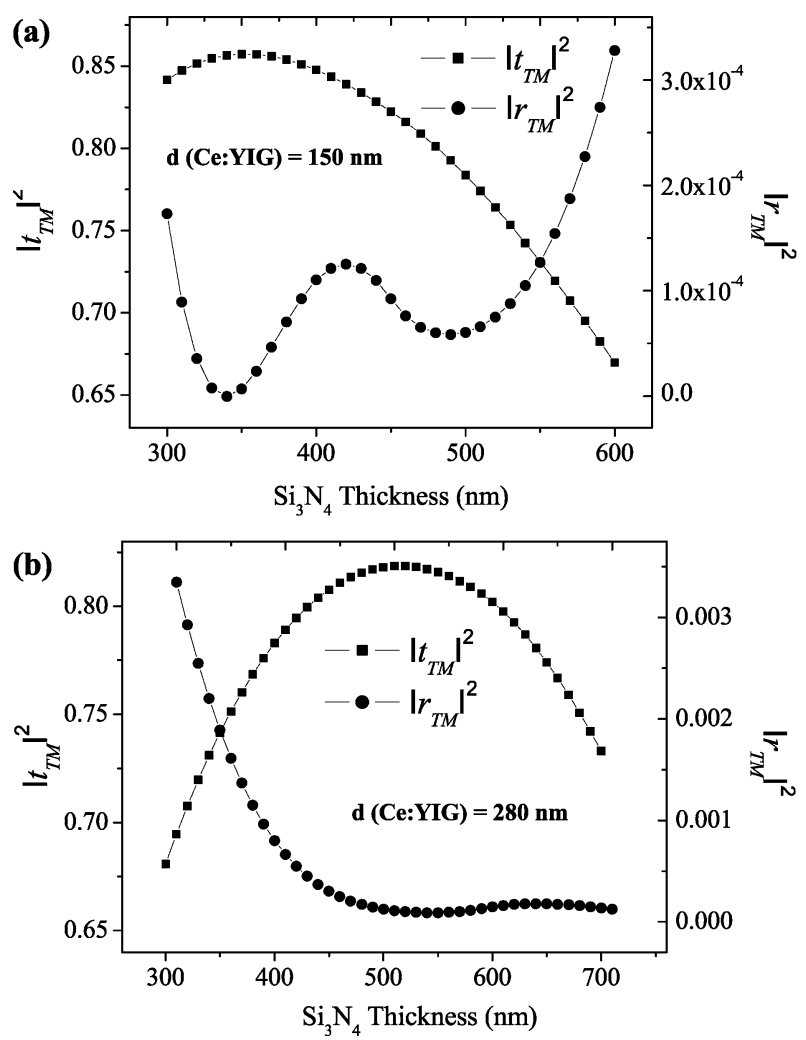

Fig. 4. (a) Transmitivity and reflectivity, respectively, for the TM mode versus the $\mathrm{Si}_{3} \mathrm{~N}_{4}$ thickness of the first and third waveguides, for a Ce:YIG thickness of $150 \mathrm{~nm}$. (b) The same as (a) but for a 280-nm Ce:YIG layer.

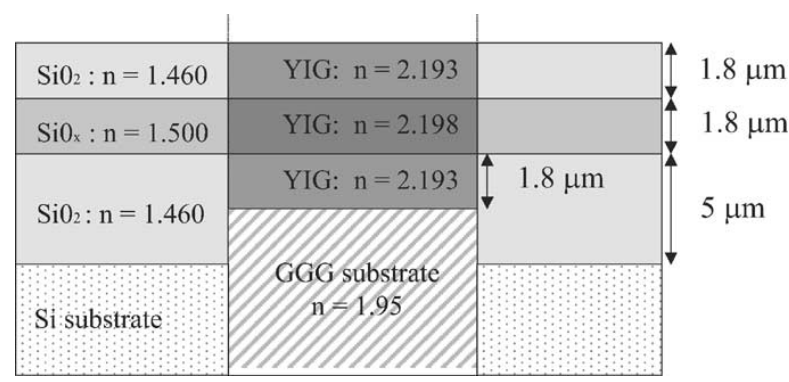

Fig. 5. Magnetooptic waveguide hybridly integrated between two silica waveguides. The configuration has been selected to obtain a $45^{\circ}$ nonreciprocal rotation.

This waveguide is hybridly integrated between two silica waveguides, as can be seen in Fig. 5.

If the discontinuities are not considered, the polarization state for an incident TE mode as a function of the length of the magnetooptic waveguide is represented in Fig. 6. These calculations are performed replacing all the coupling matrices by the identity matrix in (39).

The state of rotation and the ellipticity have a periodic dependence with the propagation distance due to the birefringence between modes. The period is $7700 \mu \mathrm{m}$, corresponding to a birefringence of

$$
N_{1}-N_{2}=1.8 \cdot 10^{-4} .
$$

On the other hand, Fig. 7 shows the polarization state of the transmitted light as a function of the length of the magnetoop- 


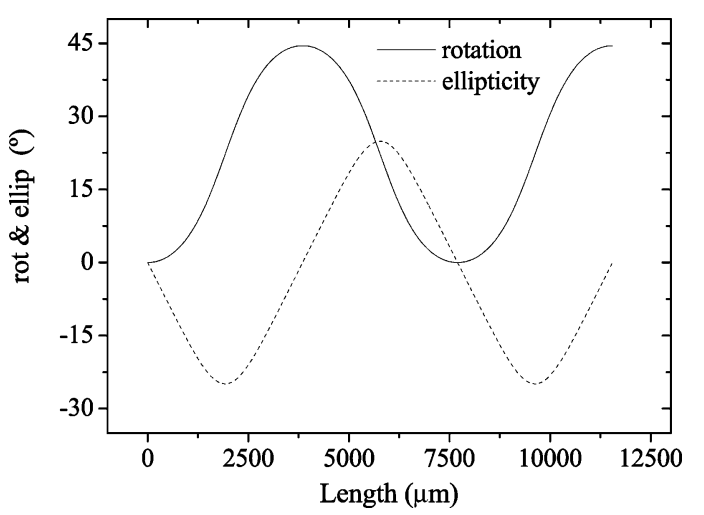

Fig. 6. Rotation and ellipticity, respectively, of the light as a function of the length of the magnetooptic waveguide when the discontinuities are not considered.

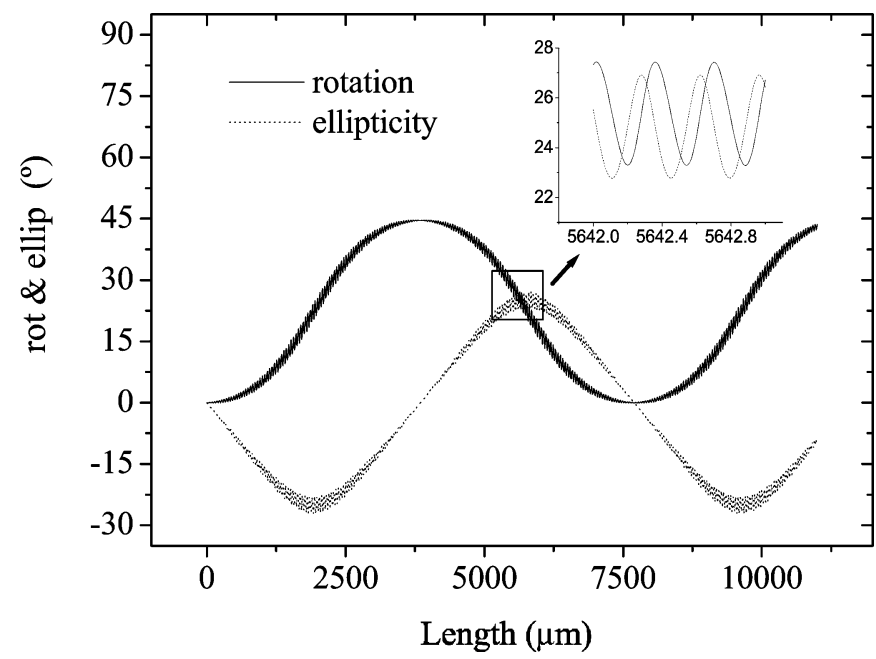

Fig. 7. Same as in Fig. 6 when the discontinuities are introduced in the calculations. The inset shows the short-period oscillations of the rotation and ellipticity as a consequence of the multiple reflections at the discontinuities.

tical waveguide, if the effect of the discontinuities is considered by means of (39)-(43).

The introduction of the discontinuities induces a new periodic effect for the reflected and transmitted light, as can be seen in the inset of Fig. 7, due to the multiple reflections at the discontinuities. As a consequence, its origin is not magnetic, and the period is given by

$$
2 d=\frac{\lambda}{N_{\mathrm{TE}}} \Rightarrow d=0.37 \mu \mathrm{m} .
$$

The reflectivity of the incident TE mode exhibits such periodic dependence, as is illustrated in Fig. 8. The high contrast between the effective propagation indexes in this hybrid configuration induces a very high values of reflectivity, as Fig. 8 shows. However, the effect of the multiple reflections can be used to diminish the reflectivity.

Therefore, the length of the second waveguide should be selected to act like an antireflecting coating for obtaining a perfect isolation. As an example, Fig. 9 shows the isolation that is achieved for the backward-traveling light in the situations in which the reflection at the discontinuities is minimized or maximized.

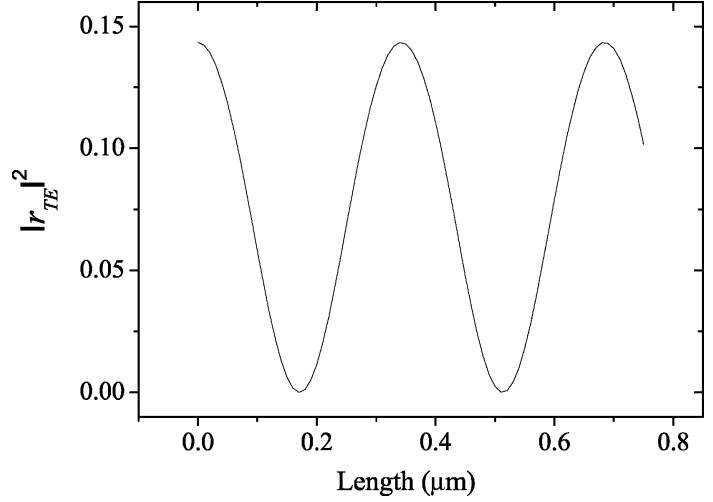

Fig. 8. Reflectivity of the TE mode versus the length of the magnetooptic guiding medium due to the effect of the multiple reflections at the discontinuities.

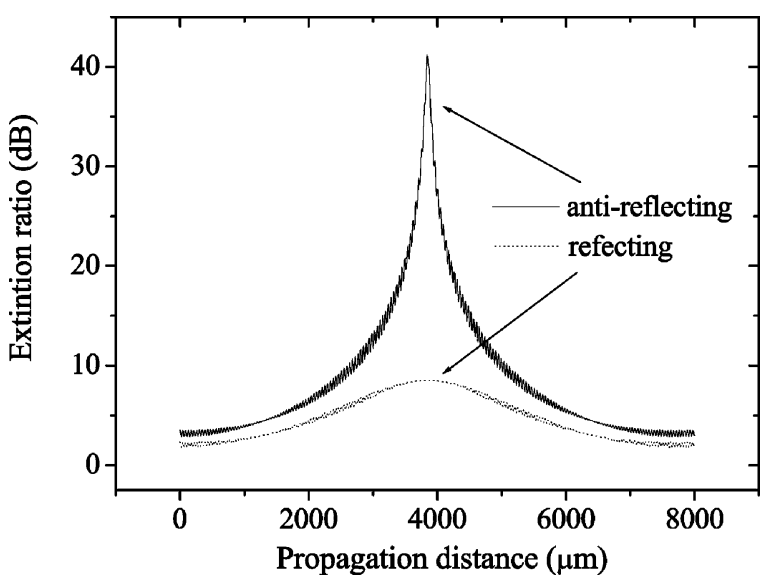

Fig. 9. Isolation of the backward-traveling light when the magnetooptic medium is in the reflecting or antireflecting configurations, respectively.

Extinction ratios as poor as $7 \mathrm{~dB}$ are obtained if the configuration is not antireflecting, as can be observed in Fig. 9. If the waveguide length is optimized, however, isolations greater than $30 \mathrm{~dB}$ can be achieved. Finally, the coupling losses at the discontinuities are $0.3 \mathrm{~dB}$ in this configuration.

Although the hybrid integrated devices can be designed to present very low coupling losses, the discontinuities play an important role, since the contrast between the effective propagation indexes in the different components can be very high.

Both examples evidence the importance of a careful design of the parameters of the integrated magnetooptic devices.

\section{CONCLUSION}

A formalism that allows the treatment of discontinuities in nonreciprocal waveguides has been presented. This formalism is based on a general orthogonality relation obtained from the reciprocity theorem, together with the modal expansion of the transverse electromagnetic fields and the continuity of such fields at both sides of the discontinuity. Finally, the formalism is expressed in a matrix form, allowing the study of the effect of several discontinuities as a simple summation and multiplication of coupling and propagations matrices. Thus, the transmitivity and reflectivity through the discontinuities is obtained straightforwardly as a solution of a set of linear equations. 
The most common nonreciprocal media are the magnetooptic media. Within this framework, examples of the presented formalism for discontinuities in magnetooptic waveguides with different orientations of the magnetization have been shown. In addition, two practical applications of the magnetooptical waveguides as isolators have been analyzed.

\section{ACKNOWLEDGMENT}

The authors would like to thank Prof. Armelles for the reading of the manuscript, suggestions, and fruitful discussions.

\section{REFERENCES}

[1] H. Dammann, E. Pross, G. Rabe, W. Tolksdorf, and M. Zinke, "Phase matching in symmetrical single-mode magneto-optic waveguides by application of stress," Appl. Phys. Lett., vol. 49, pp. 1755-1757, 1986.

[2] R. Wolfe, R. A. Lieberman, V. J. Fratello, R. E. Scotti, and N. Kopylov, "Etch-tuned ridge waveguide magneto-optic isolator," Appl. Phys. Lett., vol. 56, pp. 426-428, 1990

[3] H. Dammann, E. Pross, G. Rabe, and W. Tolksdorf, " $45^{\circ}$ waveguide isolators with phase mismatch," Appl. Phys. Lett., vol. 56, pp. 1302-1304, 1990.

[4] N. Sugimoto, H. Terui, A. Tate, Y. Katoh, Y. Yamada, A. Sugita, A. Shibukawa, and Y. Inoue, "A hybrid integrated waveguide isolator on a silica-based planar lightwave circuit," J. Lightwave Technol., vol. 14, pp. 2537-2546, Nov. 1996.

[5] K. Ando, T. Okoshi, and N. Koshizuka, "Waveguide magneto-optic isolator fabricated by laser annealing," Appl. Phys. Lett., vol. 53, pp. 4-6, 1988.

[6] T. Shintaku, "Integrated optical isolator based on efficient nonreciprocal radiation mode conversion," Appl. Phys. Lett., vol. 73, pp. 1946-1948, 1998.

[7] T. Mizumoto, S. Mashimo, T. Ida, and Y. Naito, "In-plane magnetized rare earth iron garnet for a waveguide optical isolator employing nonreciprocal phase shift," IEEE Trans. Magn., vol. 29, pp. 3417-3419, Nov. 1993.

[8] J. Fujita, M. Levy, and R. M. Osgood Jr, "Waveguide optical isolator based on Mach-Zehnder interferometer," Appl. Phys. Lett., vol. 76, pp. 2158-2160, 2000.

[9] M. Fehndrich, A. Josef, L. Wilkens, J. Kleine-Börger, N. Bahlmann, M. Lohmeyer, P. Hertel, and H. Dötsch, "Experimental investigation of the nonreciprocal phase shift os a transverse electric mode in a magnetooptic rib waveguide," Appl. Phys. Lett., vol. 74, pp. 2918-2920, 1999.

[10] V. K. Agrawal, Y. Miyazaki, and Y. Akao, "Waveguide type optical modulator using Kerr magnetooptic effects in Ni-Fe thin films: Experimental study," Jpn. J. Appl. Phys., vol. 14, pp. 1313-1322, 1975.

[11] S. E. Irvine and A. Y. Elezzabi, "Wideband magneto-optic modulation in a bismuth-substituted yttrium iron garnet waveguide," Opt. Commun., vol. 220, pp. 325-329, 2003.

[12] — "A miniature broadband bismuth-substituted yttrium iron garnet magneto-optic modulator," J. Phys. D, Appl. Phys., vol. 36, pp. 2218-2221, 2003.

[13] M. O. Vasel, "Structure of optical guided modes in planar multilayers of optically anisotropic materials," J. Opt. Soc. Amer, vol. 64, pp. 166-173, 1974.

[14] J. M. Liu and L. Gomelsky, "Vectorial beam propagation method," $J$. Opt. Soc. Amer. A, Opt. Image Sci., vol. 9, pp. 1574-1585, 1992.
[15] A. Erdmann, M. Shamonin, P. Hertel, and H. Dötsch, "Finite difference analysis of gyrotropic waveguides," Opt. Commun., vol. 102, pp. 25-30, 1993.

[16] N. Bahlmann, M. Lohmeyer, H. Dötsch, and P. Hertel, "Finite-element analysis of nonreciprocal phase shift for TE modes in magnetooptic rib waveguides with a compensation wall," IEEE J. Quantum. Electron., vol. 35, pp. 250-253, Feb. 1999.

[17] M. Lohmeyer, N. Bahlmann, O. Zhuromsky, H. Dötsch, and P. Hertel, "Phase-matched rectangular magnetooptic waveguides for applications in integrated optics isolators: Numerical assessment," Opt. Commun., vol. 158, pp. 189-200, 1998 .

[18] Y. Tsuji, M. Koshiba, and N. Takimoto, "Finite element beam propagation method for anisotropic optical waveguides," J. Lightwave Technol., vol. 17, pp. 723-728, Apr. 1999.

[19] S. Selleri, L. Vcetti, and M. Zoboli, "Full-vector finite-element beam propagation method for anisotropic optical device analysis," IEEE J. Quantum Electron., vol. 36, pp. 1392-1401, Dec. 2000.

[20] P. J. B. Clarricoats and A. B. Sharpe, "Modal matching applied to a discontinuity in planar surface waveguide," Electron. Lett., vol. 8, pp. $28-29,1972$.

[21] G. H. Brooke and M. M. Karadly, "Step discontinuities on dielectric waveguides," Electron. Lett., vol. 12, pp. 473-475, 1976.

[22] T. Rozzi, "Rigorous analysis of the step discontinuity in a planar dielectric waveguide," IEEE Trans. Microwave Theory Tech., vol. MTT-26, pp. 738-746, Oct. 1978.

[23] Q. Liu and W. C. Chew, "Analysis of discontinuities in planar dielectric waveguides: An eigenmode propagation method," IEEE Trans. Microwave Theory Tech., vol. 39, pp. 422-430, Mar. 1991.

[24] R. E. Collin, Field Theory of Guided Waves. New York: MacGrawHill, 1960.

[25] P. R. McIsaac, "A general reciprocity theorem," IEEE Trans. Microwave Theory Tech., vol. MTT-27, pp. 340-342, Apr. 1979.

[26] - "Mode orthogonality in reciprocal and nonreciprocal waveguides," IEEE Trans. Microwave Theory Tech., vol. 38, pp. 1808-1816, Nov. 1991.

[27] T. Tamir et al., Guided-Wave Optoelectronic. New York: SpringerVerlag, 1988

[28] A. K. Zvezdin and V. A. Kotov, Modern Magnetooptic and Magnetooptical Materials. Bristol, U.K.: IOP Publishing, 1997.

[29] B. Sepúlveda, Y. Huttel, C. Martínez Boubeta, A. Cebollada, and G Armelles, "Linear and quadratic magneto-optical Kerr effects in continuous and granular ultrathin monocrystalline Fe films," Phys. Rev. B, Condens. Matter, vol. 68, p. 064 401, 2003.

[30] B. Stadler and A. Gopinath, "Magneto-optical garnet films made by reactive sputtering," IEEE Trans. Magn., vol. 36, pp. 3957-3961, Nov. 2000

[31] T. Uno and S. Noge, "Growth of magneto-optic Ce:YIG thin films on amorphous silica substrates," J. Eur. Ceramic Soc., vol. 21, pp. 1957-1960, 2001

Borja Sepúlveda, photograph and biography not available at the time of publication.

Laura M. Lechuga, photograph and biography not available at the time of publication. 